\title{
Natural history of aspirin-induced asthma
}

\author{
A. Szczeklik, E. Nizankowska, M. Duplaga, on behalf of the AIANE Investigators
}

\begin{abstract}
Natural history of aspirin-induced asthma. A. Szczeklik, E. Niżankowska, M. Duplaga for the AIANE Investigators. C) ERS Journals Ltd 2000.

ABSTRACT: There is a subset of patients with bronchial asthma who are susceptible to disease exacerbation upon receiving aspirin and other nonsteroidal anti-inflammatory drugs. This is a clinical syndrome, called aspirin-induced asthma (AIA), associated with alterations in arachidonate metabolism and cysteinyl-leukotriene overproduction. The natural history and clinical characteristics of this type of asthma were studied.
\end{abstract}

Sixteen clinical centres in $\mathbf{1 0}$ European countries provided standardized information to the specially developed patient-oriented database regarding: medical history, physical examination, diagnosis, and treatment. Diagnosis of AIA was based on a typical history, confirmed by positive aspirin provocation tests, carried out in $\mathbf{9 1 \%}$ of the patients. A total of 500 patients were enrolled in the study.

AIA developed according to a pattern, characterized by a sequence of symptoms. First, persistent rhinitis, appearing at a mean age of $29.7 \pm 12.5 \mathrm{yrs}$, then asthma, aspirin intolerance and nasal polyposis appear. The clinical presentation in different European countries was remarkably similar. In females, who outnumbered males by 2.3:1, the onset of symptoms occurred significantly earlier and the disease was more progressive and severe than in males. Atopy, present in approximately a third of patients, led to earlier manifestation of rhinitis and asthma, but not of aspirin intolerance or nasal polyposis. A family history of aspirin intolerance, recorded in $6 \%$ of patients, had a less evident effect on the course of the disease than sex or atopy. Fifty one per cent of patients, in addition to inhaled steroids, required chronic systemic corticosteroid therapy at a mean dose of $8 \mathrm{mg}^{\text {prednisone. day }}{ }^{-1}$. Surprisingly, $15 \%$ of patients were unaware of intolerance to aspirin and learnt about it only after having provocation tests performed.

All over Europe, aspirin-induced asthma develops in a similar characteristic way. Its course is influenced by sex and the presence of atopy. In half of the patients, asthma is severe, and steroid-dependent. The uniform natural history of aspirin-induced asthma might suggest a common underlying principle.

Eur Respir J 2000; 16: 432-436.
Dept of Medicine, Jagellonian University School of Medicine, Cracow, Poland.

Correspondence: A. Szczeklik

Jagellonian University School of Medicine Dept of Medicine

Skawiñska 8

31-066 Cracow

Poland

Fax: 48124305203.

Keywords: Aspirin

aspirin-induced asthma

bronchial asthma

eicosanoids

eosinophil

leukotrienes

Received: January 312000

Accepted after revision April 252000

This study was supported by the European Commission, Directorate General for Science, Research and Development; the Polish State Research Council; the Swedish Heart Lung Foundation; the Swedish Association Against Asthma \& Allergy and the Swedish Foundation for Health Care Sciences \& Allergy Research.
Bronchial asthma, one of the most common diseases, is not a homogenous entity. It might have different causes, which, acting against a predisposing genetic background, lead through various routes to the final clinical picture of dyspnoea, often paroxysmal, due to reversible bronchial obturation. Several types of asthma have been distinguished, depending on the criteria used, e.g. presence of atopy (atopic and nonatopic), season (pollen versus perennial), precipitating factors (exercise and industrial), severity of disease (sporadic and severe) and response to treatment (steroid-dependent and steroid-resistant). Clearcut differentiation amongst these types is not always possible and overlap can occur [1].

Aspirin-induced asthma (AIA) appears to be a distinct clinical syndrome [2]. Its hallmark is precipitation of asthmatic attacks by aspirin and other nonsteroidal antiinflammatory drugs (NSAIDs) which inhibit cyclo-oxygenase $[3,4]$. The first reports of violent, sometimes fatal, attacks of asthma following ingestion of aspirin appeared shortly after introduction of the drug to therapy. The association of aspirin sensitivity, asthma and nasal polyposis was described by WIDAL et al. [5], in 1922, and this clinical entity, subsequently named aspirin triad, was popularized by the studies of SAMTER and BEERS [6] in the late $1960 \mathrm{~s}$. The incidence of AIA in the normal population is $0.3-0.6 \%[7,8]$. In adult asthmatics, it ranges $3-21 \%$ depending on the diagnostic methods used. When aspirin challenge coupled with spirometry is performed, the frequency among adult asthmatics is $8-20 \%$, whereas surveys relying on history alone have reported a lower frequency, usually $\sim 5 \%$ [7-10]. Recent interest has been stirred by the finding in AIA of alterations in arachidonate metabolic pathways [11-15], leading to cysteinyl-leukotriene overproduction [16-18]. The availability of antileukotriene drugs has amplified interest in this syndrome $[19,20]$. The natural history and clinical picture of AIA, based on a large European survey, are presented here.

\section{Design of the study and methods}

Sixteen clinical centres in 10 European countries, participating in the European Network on Aspirin-Induced Asthma (AIANE), took part in the study. They collected 
data, using a unified questionnaire, developed with the software MS Access 1.1 (Statsoft Inc., Tulsa, USA). The patient-oriented database was developed during two international workshops held in Cracow, Poland, and included detailed medical history, physical examination results, diagnostic methods and treatment. Asthma was diagnosed according to the criteria of the American Thoracic Society [21]. The diagnosis of aspirin intolerance was based on a typical history, confirmed by positive aspirin provocation tests, which were carried out in $91 \%$ of patients. The bronchial provocation test [22] with increasing doses of inhaled L-lysine-aspirin (Aspisol; Bayer AG, Leverkusen, Germany), using a standardized protocol and inhalatory dosimeters (Spira Electro 2, Spira Respiratory Care Center Ltd., Hämeelinna, Finland) provided through the Network, was that most commonly performed [23]. The test was considered positive if clinical symptoms were precipitated and the forced expiratory volume in one second (FEV1) fell by $\geq 20 \%$ from initial values as compared to placebo. In some cases, oral provocation tests with aspirin [9] were performed; the criteria for positivity were the same as in the bronchial tests. In $9 \%$ of the asthmatic patients with an unequivocal history of aspirin intolerance, testing was not carried out because of clinical instability or lack of patient consent.

In a subset of patients from two centres (Davos, Switzerland $(n=66)$ and Cracow, Poland $(n=37))$ detailed study of the presence or absence of atopy was carried out, using the same methods. These included: skin-prick tests with common aeroallergens, and determination of total serum immunoglobulin E (IgE) levels and levels of specific IgE directed against common allergens (CAP-System; Pharmacia, Uppsala, Sweden). A detailed personal and family history of atopy constituted part of the uniform questionnaire, used in all the patients participating in the study.

The data were transferred to the University Dept of Medicine in Cracow for analysis and statistical evaluation. The latter was performed using the software Statistica for Windows 5.1 (Statsoft Inc., Tulsa, USA). The differences between patients from two groups were assessed using an unpaired t-test, or, if the variable did not show normal distribution, a nonparametric test (Mann-Whitney U-test) for independent samples in case of nondiscrete values. Categorical variables were compared using the Chi-squared test and two-tailed Fisher exact test, if applicable. The differences between more than two groups of patients were determined by the analysis of variance (ANOVA) procedure for nondiscrete variables and Chi-squared test for categorical variables. The normal distribution of the variables was assessed using the Kolmogorov-Smirnov test.

\section{Results}

A total of 500 patients were enrolled in the study. They came from Bulgaria $(n=15)$, the UK $(n=13)$, France $(n=$ $12)$, Germany $(\mathrm{n}=104)$, Italy $(\mathrm{n}=38)$, Portugal $(\mathrm{n}=32)$, Poland $(n=233)$, Spain $(n=38)$ and Sweden $(n=15)$. Their basic characteristics, including sex, are presented in table 1. At enrollment, the mean \pm SD age was $45.2 \pm 12.5$ yrs (range 6.3-76.2 yrs); the lower and upper quartiles were 37.2 and 54.2 yrs, respectively. The geometric mean of the inhaled provocation dose causing a $\geq 20 \%$ fall in FEV1, was $6.1 \mathrm{mg}$ acetylsolicylic acid (range 0.2-170.0 $\mathrm{mg})$.
ANOVA of the main clinical characteristics was used to compare patients from countries which enrolled $>15$ cases, including Polish patients, who constituted the largest cohort. Patients' age as at onset of: 1) rhinitis; 2) asthma; and 3) NSAID intolerance did not differ amongst countries. Similarly, there was no difference in the sex ratio. Rhinitis was slightly more common in Spaniards (97\%), and Germans $(90 \%)$ than in patients from the remaining countries (74-87\%). The time of the first diagnosis of nasal polyposis differed amongst the countries.

Rhinitis was the first symptom of the disease, and was related to a flu-like infection in half of the patients. It appeared on average at an age of $30 \mathrm{yrs}$, was characterized by discharge from the nose, often watery, nasal blockage and sneezing, and, less frequently, by pain in paranasal sinuses. It was perennial, difficult to treat and led to loss of smell in 55\% of patients. In an average patient, 2 yrs after commencement of rhinitis, the first symptoms of asthma appeared. Intolerance to aspirin and/or other NSAIDs became evident 4 yrs later (fig. 1). Nasal polyps were diagnosed at about the same time in $60 \%$ of subjects. There was a close linear association between mean age at onset of rhinitis, asthma, NSAID intolerance and nasal polyps (correlation coefficient for each pair of variables $>0.75$, $\mathrm{p}<0.05)$.

Factors precipitating the first attack of asthma were upper respiratory infection (45\%), intake of aspirin and/or NSAIDs (14\%), allergen or industrial exposure (11\%) and unknown $(30 \%)$.

Aspirin was the NSAID which precipitated the most adverse reactions ( $82 \%$ of patients), followed by pyrazolones $(9 \%)$. The triggered symptoms included dyspnoea $(88 \%)$, nasal discharge and blockage $(42 \%)$, skin manifestations, i.e. urticaria or scarlet flush (20\%), conjunctival irritation (15\%), angio-oedema $(8 \%)$ and anaphylactoid shock with hypotension and loss of consciousness (6\%). It is of interest that $15 \%$ of patients were unaware of intolerance to aspirin, and became conscious of it only after provocation tests were performed. In these cases, the clues from the history which prompted the physician to perform a provocation test were nasal polyps and/or sinus disease. Adverse reactions to antibiotics were reported by $18 \%$ of patients, usually with skin or gastrointestinal manifestation.

Inhaled corticosteroids were used for chronic asthma treatment in $80 \%$ of patients and oral in $51 \%$. The latter were administered for control of asthma for, on average, $7.5 \mathrm{yrs}$ at a dose corresponding to $8 \mathrm{mg}$ prednisone $\cdot$ day $^{-1}$. Twenty four per cent of patients received intravenous corticosteroids during the 12 months preceding registration in the AIANE database. The side-effects of systemic corticosteroids included obesity (16\%), osteoporosis (13\%) and arterial hypertension (9\%). The frequency of therapeutic aspirin desensitization varied greatly among the participating centres. It was commonly performed in Davos and Rome, but infrequently in the other centres.

Females outnumbered males by $2.3: 1$. The symptoms of disease emerged earlier in females than in males. Both rhinitis and aspirin intolerance appeared on average 3 yrs earlier in females compared to males, and these differences were significant. Although the duration and dosing of oral corticosteroid therapy were similar in both sexes, asthma was more poorly controlled in females, as evidenced by the 
Table 1. - Characteristics of patients enrolled in study

\begin{tabular}{|c|c|c|c|c|}
\hline & Total & Females & Males & p-value \\
\hline Patients $n$ & 500 & 348 & 152 & \\
\hline Age at registration in database yrs & $45.2 \pm 12.5$ & $44.9 \pm 12.1$ & $45.9 \pm 13.4$ & $\mathrm{NS}^{*}$ \\
\hline Age at first symptoms of rhinitis yrs & $29.7 \pm 12.5$ & $28.8 \pm 12.0$ & $31.8 \pm 13.3$ & $0.02 *$ \\
\hline Age at first symptoms of asthma yrs & $31.9 \pm 13.5$ & $31.4 \pm 13.0$ & $33.2 \pm 14.6$ & NS* \\
\hline Age at nasal polyps diagnosis yrs & $35.2 \pm 12.13$ & $34.8 \pm 12.0$ & $35.9 \pm 12.4$ & $\mathrm{NS}^{*}$ \\
\hline Age at first aspirin intolerance symptoms yrs & $35.2 \pm 12.5$ & $34.4 \pm 12.3$ & $37.3 \pm 12.5$ & $0.02 *$ \\
\hline Occurrence of rhinitis \% & 82.4 & 81.3 & 84.9 & $\mathrm{NS}^{+}$ \\
\hline Occurrence of nasal polyps $\%$ & 60.4 & 56.0 & 70.4 & $0.003^{+}$ \\
\hline Polypectomies $\mathrm{n}$ & $2.6 \pm 3.1$ & $2.4 \pm 2.7$ & $3.0 \pm 3.6$ & $\mathrm{NS}^{\#}$ \\
\hline Positive family history of aspirin intolerance $\%$ & 5.8 & 6.9 & 3.3 & $\mathrm{NS}^{+}$ \\
\hline Chronic oral corticosteroid therapy $\%$ & 51.4 & 54.6 & 44.1 & $0.03^{+}$ \\
\hline Duration of oral corticosteroid therapy yrs & $7.5 \pm 5.5$ & $7.55 \pm 5.5$ & $7.5 \pm 5.5$ & NS* \\
\hline Prednisone dose $\mathrm{\xi}^{\S}$ & $7.7 \pm 6.5$ & $7.8 \pm 6.8$ & $7.2 \pm 5.5$ & NS* \\
\hline Hospitalizations for asthma exacerbations ${ }^{\S} \mathrm{n}$ & $0.6 \pm 1.4$ & $0.8 \pm 1.6$ & $0.3 \pm 1.0$ & $0.006^{\#}$ \\
\hline Emergency interventions ${ }^{\S} \mathrm{n}$ & $1.3 \pm 2.7$ & $1.7 \pm 3.0$ & $0.6 \pm 1.3$ & $0.001^{\#}$ \\
\hline
\end{tabular}

Data are presented as mean $\pm \mathrm{SD}$, absolute number or percentages. ${ }^{\S}$ : in 12 months preceding registration; *: t-test for independent samples; : Mann-Whitney U-test; ${ }^{+}$: Pearson Chi-squared test. NS: nonsignificant.

significantly higher number of emergency interventions and hospital admissions for asthma exacerbations (table 1).

Familial history of intolerance to aspirin was reported by $6 \%$ of the patients. These patients showed a tendency to earlier appearance of symptoms of aspirin intolerance (31.0土 13.3 versus $35.5 \pm 11.2 \mathrm{yrs} ; \mathrm{p}=0.09)$ and earlier diagnosis of nasal polyps $(30.2 \pm 14.4$ versus $35.5 \pm 12.0 \mathrm{yrs}$; $\mathrm{p}=0.08)$. The family history did not influence severity of asthma.

Common concomitant diseases were recorded at the expected frequency. Churg-Strauss syndrome (CSS), confirmed by biopsy, was diagnosed in five patients. Three of these received no antileukotriene drugs; in the remaining two, symptoms of CSS appeared during treatment with leukotriene receptor antagonists coupled with a reduction in the steroid dose. An attempt to distinguish the natural prevalence of CSS in asthma sufferers suggested a background of 36-80 cases of CSS per million asthmatics per yr [24].

In a group of 103 German and Polish patients, a standardized search for atopy revealed a positive skin-prick test to at least one common allergen in $34 \%$, total serum IgE levels of $>100 \mathrm{IU} \cdot \mathrm{mL}^{-1}$ in $46 \%$ (geometric mean for the whole group $87.2 \mathrm{IU} \cdot \mathrm{mL}^{-1}$ ), and levels of specific serum IgE directed against common allergens (class $\geq 2.0$ )

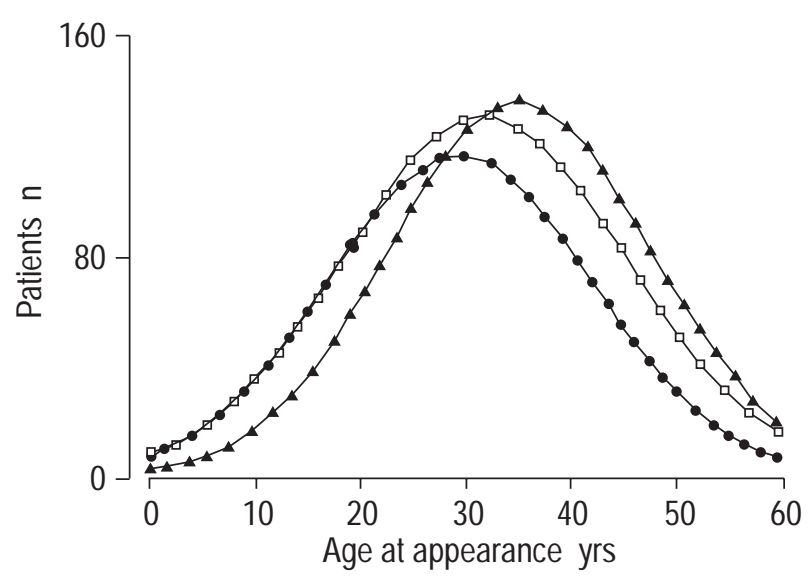

Fig. 1. - Distributions of appearance of the first symptoms of rhinitis $(\mathbf{O})$, asthma $(\square)$ and aspirin intolerance $(\boldsymbol{\Delta})$. in $31 \%$. Comparison of patients with positive and negative skin tests disclosed that rhinitis appeared 6 yrs earlier (23.0 \pm 11.1 versus $28.9 \pm 10.6 \mathrm{yrs} ; \mathrm{p}<0.02)$ and asthma $7 \mathrm{yrs}$ earlier $(25.0 \pm 10.9$ versus $32.1 \pm 9.7 \mathrm{yrs} ; \mathrm{p}<0.02)$ in the former as compared to the latter. However, the mean age at which either aspirin intolerance emerged or nasal polyps were diagnosed did not differ between the two groups. Statistical analysis revealed that the effect of atopy on the course of the disease was independent of the effect of sex.

\section{Discussion}

The clinical syndrome, studied here, develops according to a pattern, characterized by a sequence of symptoms. First, persistent rhinitis, then asthma, aspirin intolerance and nasal polyposis appear. The time of the first diagnosis of nasal polyps was variable; it depended very much on use of rhinoscopic examination. Therefore, its place in the sequence of emerging symptoms might be debatable. Patients' subjective assessment and memory could have affected the reported order of symptoms. Although, in individual patients, some variations occur, in the whole population of patients, there was a close association between age and order of appearance of the main symptoms. These results correspond to observations made previously by clinicians on much smaller groups of patients confined to one centre $[6,9,25]$. In this respect, similarities in clinical presentation between different European countries are remarkable. Since the methodology used in the present study has been strictly standardized, it may be concluded that AIA develops according to a similar pattern all over Europe. This might suggest a common underlying principle [26]. Some variations in time of diagnosis of nasal polyps or aspirin intolerance could be explained by local differences in performing routine rhinoscopy or patients awareness of appearance of intolerance to a drug they have been taking safely for many years.

Three factors exerted influence on the natural history of AIA: 1) sex, 2) atopy, and 3) family history of aspirin intolerance.

The predominance of AIA among females has been observed previously [5, 6, 9,27], although the effect of sex 
on the course of the disease has not been studied. The present data clearly indicates that the disease differs in presentation between females and males. Not only does the onset of symptoms occur earlier in females, but females tend also to exhibit a more progressive and severe disease than males. Sex hormones may modulate susceptibility by affecting immune response and repair mechanisms [28]. In autoimmune disease, such as multiple sclerosis or rheumatoid arthritis, the female to male ratio is $2: 1-3: 1$, whereas, in lupus, the distribution is more skewed, with nine times as many females affected as males [28]. It is, therefore, interesting to note that a proportion of patients with AIA show laboratory markers of autoimmunity and clinical manifestation of autoimmune phenomena, although of weak intensity [29]. Irrespective of the underlying reasons, sex differences during the course of AIA have implications for management of patients. Perhaps, there are sex-specific effects in response to therapy as well as to disease.

Early studies [6, 23], performed before the advent of serum IgE determinations, led to widespread belief that AIA belongs to intrinsic asthma, and is observed rarely in atopic subjects. This view has been questioned recently [30]. The present data clearly demonstrates that approximately one third of AIA patients exhibit both clinical and immunological markers of atopy. This is close to prevalence of atopy reported in large community surveys in European adults [31, 32]. In a recent epidemiological study in Finland [8], the risk of aspirin intolerance causing asthma was eight times higher in people with allergic rhinitis than in those without it. Similarly, in Turkey [10], allergic conditions and serum IgE levels were higher in asthmatic patients with aspirin intolerance than in those who tolerated aspirin well. In the present study, the group of atopic patients experienced symptoms of rhinitis and asthma earlier than nonatopic ones, but aspirin-intolerance and nasal polyps were diagnosed at the same age in both groups. This is in accordance with the succinct remark of SAMTER and BEERS [6] that, in a patient with clinical signs of atopy, development of aspirin intolerance does not indicate another sensitivity, but marks an entirely new, profound disease, superseding the atopic state. A family history of aspirin intolerance, present in $6 \%$ of the patients in the present study, seemed to have less effect on the course of the disease than sex or atopy. In patients with positive family history, aspirin intolerance and nasal polyps tended to be diagnosed earlier, but the severity of asthma was unaffected.

Once developed, asthma runs a protracted course, despite avoidance of aspirin and cross-reacting drugs [2]. This type of asthma is not easy to treat. Half of the patients in the present study, in addition to inhaled steroids, required chronic oral corticosteroid therapy, a quarter received emergency intravenous corticosteroids treatment during the year preceding registration in the database. It is remarkable that $15 \%$ of patients were completely unaware of being aspirin-intolerant and realized it only after performance of provocation tests. This result supports the recent opinion [33] that aspirin intolerance is underdiagnosed within the asthmatic population. According to SAMPSON [33], the reasons for underreporting of aspirin sensitivity may include the deliberate avoidance of NSAIDs by asthmatics aware of the risk of adverse reactions, or a lack of recognition by patients of mild
NSAID-induced reactions because of their delayed onset of action. Underdiagnosis of aspirin sensitivity may be due to the lack of routine aspirin challenge testing of asthmatic patients who do not report a positive history of aspirin sensitivity. Intolerance to aspirin can be masked by such drugs as corticosteroids or long-acting $\beta_{2}$-mimetics [2]. Underreporting and underdiagnosis of aspirin sensitivity may have catastrophic consequences for patients. In a large survey [34] $25 \%$ of asthmatic patients requiring emergency mechanical ventilation were found to have AIA. The authors' experience demonstrates that aspirin challenge, especially by the inhalational route, is a safe and reliable procedure. Its diagnostic sensitivity and specificity is similar to that of the oral provocation test [35]. It deserves to be performed more frequently in order to diagnose the relatively common and distinct type of asthma described here.

Participating investigators of AIANE (European Network of Aspirin-Induced Asthma). Bulgaria: T.A. Popov, Clinical Centre of Allergology, Sophia; France: J. Bousquet, P. Godard, Service des Maladies Respiratoires, Hôpital Arnaud de Villeneuvre, Montpellier; M. Joseph, Institut Pasteur, Lille; D. Vervloet, Centre Hospitalier Regional et Universitaire, Dept des Maladies Respiratories, Hôpital Ste Marguerite, Marseille; Germany: L. Jäger, A. Machnik, FSU Jena, Medical Faculty, Institute of Clinical Immunology, Jena; G. Kunkel, J. Niechus, Dept of Clinical Immunology and Asthma Polyclinic, Berlin; Italy: S. Bianco, M. Robuschi, University of Milan, Nuovo Ospedale San Gerardo, Div. Pneumologie, MonzoMilano; G. Patriarca, C. D'Ambrosio, D. Schavino, E. Nucera, Università Cattolica del Sacro Cuore, Policlinico A. Gemelli, Servizio di Allergologia, Rome; Poland: M.L. Kowalski, B. Bieñkiewicz, Medical Academy, Dept of Immunology, Lódż; J. Rożniecki, P. Kuna, Medical Academy. Dept of Pneumonology and Allergology, Lódż; G. Bochenek, Dept of Medicine, Jagellonian University School of Medicine, Cracow; Portugal: M. Vaz Azevedo, J. Rodrigues, E. Faria, Faculty of Medicine, Unidade de Imunoalergologia, Hospital de S. Joãó, Porto; Spain: C. Picado, J. Mullol, J. Casadevall, University of Barcelona, Facultat de Medicina, Hospital Clinic i Provincial, Division of Pneumology and Respiratory Allergy, Barcelona; Sweden: B. Dahlén, S-E. Dahlén, Karolinska Institutet, Dept of Thoracic Medicine, Stockholm; Switzerland: M. Schmitz-Schumann, Hochgebirgsklinik Davos-Wolfgang, Dept Pneumologie I Asthma und Allergieklinik, Davos; UK: T.H. Lee, University of London, King's College, Guy's Hospital, Respiratory Medicine and Allergy, London.

Acknowledgements: The authors thank R. Pauwels for help in the initial stage of the project.

\section{References}

1. Barnes PJ. Pathophysiology of asthma. In: Barnes PJ, Rodger IW, Thomson NC, eds. Asthma Basic Mechanism and Clinical Management. London, Academic Press, 1998; pp. 487-506.

2. Szczeklik A, Stevenson DD. Aspirin-induced asthma: advances in pathogenesis and management. J Allergy Clin Immunol 1999; 104: 5-13. 
3. Szczeklik A, Gryglewski RJ, Czerniawska-Mysik G. Relationship of inhibition of prostaglandin biosynthesis by analgesics to asthma attacks in aspirin-sensitive patients. BMJ 1975; 1: 67-69.

4. Lee TH. Mechanism of aspirin sensitivity. Am Rev Respir Dis 1992; 145: 34-36.

5. Widal MF, Abrami P, Lermoyez J. Anaphylaxie et idiosyncrasie. Presse Med 1922; 30: 189-192.

6. Samter M, Beers RF. Intolerance to aspirin. Clinical studies and consideration of its pathogenesis. Ann Int Med 1968; 68: 975-983.

7. Settipane GA, Chafee FH, Klein DE. Aspirin intolerance. II. A prospective study in an atopic and normal population. J Allergy Clin Immunol 1974; 53: 200-204.

8. Hedman J, Kaprio J, Poussa T, Nieminen MM. Prevalence of asthma, aspirin intolerance, nasal polyposis and chronic obstructive pulmonary disease in a population-based study. Int J Epidemiol 1999; 28: 717-722.

9. Stevenson DD. Diagnosis, prevention and treatment of adverse reactions to aspirin and nonsteroidal antiinflammatory drugs. J Allergy Clin Immunol 1984; 74 : 617-622.

10. Kalyoncu AF, Karakaya G, Sahin AA, Baris YI. Occurrence of allergic conditions in asthmatic with analgesic intolerance. Allergy 1999; 54: 428-435.

11. Szczeklik A, Sladek K, Dworski R, et al. Bronchial aspirin challenge causes specific eicosanoid response in aspirin sensitive asthmatics. Am J Respir Crit Care Med 1996; 154: 1608-1614.

12. Sestini P, Armetti L, Gambaro G, et al. Inhaled $\mathrm{PGE}_{2}$ prevents aspirin-induced bronchoconstriction and urinary $\mathrm{LTE}_{4}$ excretion in aspirin-sensitive asthma. Am J Respir Crit Care Med 1996; 153: 572-575.

13. Picado C, Fernandez-Morta C, Juan $\mathrm{M}$, et al. Cyclooxygenase-2 mRNA is downexpressed in nasal polyps from aspirin-sensitive asthmatics. Am $J$ Respir Crit Care Med 1999; 160: 291-296.

14. Cowburn AS, Sladek K, Soja J, et al. Overexpression of leukotriene C4 synthase in bronchial biopsies from patients with aspirin-intolerant asthma. J Clin Invest 1998; 101: 1-13.

15. Sanak M, Simon H-U, Szczeklik A. Leukotriene $\mathrm{C}_{4}$ synthase promoter polymorphism and risk of aspirininduced asthma. Lancet 1997; 350: 1599-1600.

16. Kumlin $\mathrm{M}$, Dahlen B, Bjorck $\mathrm{T}$, Zetterstrom $\mathrm{O}$, Granstrom E, Dahlen S-E. Urinary excretion of leukotriene $\mathrm{E}_{4}$ and 11-dehydro-thromboxane $\mathrm{B}_{2}$ in response to provocations with allergen, aspirin, leukotriene $\mathrm{D}_{4}$ and histamine in asthmatics. Am Rev Respir Dis 1992; 146: 96-103.

17. Kowalski ML, Sliwinska-Kowalska M, Igarashi Y, et al. Nasal secretions in response to acetylsalicylic acid. $J$ Allergy Clin Immunol 1993; 91: 580-598.

18. Nasser SMS, Lee TH. Leukotrienes in aspirin-sensitive asthma. In: Szczeklik A, Gryglewski RJ, Vane JR, eds. Eicosanoids, Aspirin and Asthma, New York, Marcel Dekker, Inc. 1998; pp. 317-335.

19. Holgate ST, Bradding P, Sampson AP. Leukotriene antagonists and synthesis inhibitors: new directions in asthma therapy. J Allergy Clin Immunol 1996; 98: 1-13.
20. Dahlen B, Nizankowska E, Szczeklik A, et al. Benefits from adding the 5-lipoxygenase inhibitor zileuton to conventional therapy in aspirin-intolerant asthmatics. $\mathrm{Am}$ J Respir Crit Care Med 1998; 157: 1187-1194.

21. American Thoracic Society. Standards for the diagnosis and care of patients with chronic obstructive pulmonary disease (COPD) and asthma. Am Rev Respir Dis 1987; 136: 225-244.

22. Bianco S, Robuschi M, Petrigni G. Aspirin-induced tolerance in aspirin-induced asthma detected by a new challenge technique. IRCS J Med Sci 1977; 5: 129-130.

23. Dahlen B, Kumlin M, Margolskee DJ, et al. The leukotriene-receptor antagonist MK-0679 blocks airway obstruction induced by inhaled lysine-aspirin in aspirin-sensitive asthmatics. Eur Respir J 1993; 6: 10181026.

24. Hart B. Replacing or reducing high dose oral corticosteroids with alternative asthma controller therapies: implications for leukotriene receptor antagonists. Eur Respir Rev 1998; 8: 1056-1058.

25. Grzelewska-Rzymowska I, Rozniecki J, Szmidt M, Kowalski ML. Astma z nadwrazliwoscia na aspiryne: obraz Kliniczny. Pol Arch Int Med 1982; 67: 7-13.

26. Szczeklik A. Aspirin-induced asthma as a viral disease. Clin Allergy 1988; 18: 15-20.

27. Giraldo M, Blumenthal N, Spink WW. Aspirin intolerance and asthma. A clinical and immunological study. Ann Intern Med 1969; 71: 479-496.

28. Whitacre CC, Reingold SC, O'Looney PA, and the Task Force on Gender, Multiple Sclerosis and Autoimmunity. A gender gap in autoimmunity. Science 1999; 283: 12771278.

29. Szczeklik A, Nizankowska E, Serafin A, Dyczek A, Duplaga M, Musial J. Autoimmune phenomena in bronchial asthma with special reference to aspirin intolerance. Am J Respir Crit Care Med 1995; 152: 1753-1756.

30. Bochenek G, Nizankowska E, Szczeklik A. Atopy trait in hypersensitivity to nonsteroidal anti-inflammatory drugs. Allergy 1996; 51: 16-23.

31. Tschopp JM, Sistek D, Schindler C, et al. Current allergic asthma and rhinitis: diagnostic efficiency of three commonly used atopic markers (IgE, skin prick tests, and Phadiatop). Allergy 1998; 53: 608-613.

32. Charpin D, Sibbald B, Week E, Wuthrich B. Epidemiologic identification of allergic rhinitis. Allergy 1996; 51: 293-298.

33. Sampson AP. Drug-induced asthma: NSAID-s, beta blockers and ACE-inhibitors. In: Holgate ST, Boushey HA, Fabbri LM, eds. Difficult Asthma, London, M. Dunitz Ltd. 1999; pp. 127-146.

34. Marquette $\mathrm{CH}$, Saulnier F, Leroy $\mathrm{O}$, et al. Long-term prognosis for near-fatal asthma. A 6-year follow-up study of 145 asthmatic patients who underwent mechanical ventilation for near-fatal attack of asthma. Am Rev Respir Dis 1992; 146: 76-81.

35. Nizankowska E, Bestynska-Krypel A, Cmiel A, Szczeklik A. Oral and bronchial provocation tests with aspirin for diagnosis of aspirin-induced asthma. Eur Respir J 2000; 15: 863-869. 